${ }^{1}$ Centro de Información Toxicológica Pontificia Universidad Católica de Chile (CITUC).

${ }^{2}$ Departamento de Farmacia, Facultad de Química, Pontificia Universidad Católica de Chile. 'Departamento de Laboratorios Clínicos, Escuela de Medicina, Pontificia Universidad Católica de Chile.

Recibido el 6 de marzo de 2015, aceptado el 13 de agosto de 2015.

Correspondencia a:

Dr. Juan Carlos Ríos Centro de Información Toxicológica y de Medicamentos, Escuela de Medicina de la Pontificia Universidad Católica de Chile (CITUC). Marcoleta 446. Santiago Centro, Santiago. Teléfono: (56-2) 3543194. Fax: (56-2) 2472112. jcrios@med.puc.cl

\section{Caracterización de las exposiciones a plaguicidas entre los años 2006 y 2013 reportadas al Centro de Información Toxicológica de la Pontificia Universidad Católica de Chile}

\author{
WALDO GUTIÉRREZ ${ }^{1}$, PATRICIA CERDA ${ }^{1}$, JOSÉ CRISTIAN PLAZA-PLAZA ${ }^{2}$,
} JUAN JOSÉ MIERES ${ }^{1}$, ENRIQUE PARIS ${ }^{1}$, JUAN CARLOS RÍOS ${ }^{1,3}$

\section{Characterization of pesticide exposures reported between 2006 and 2013 to a poison information center in Chile}

Background: Pesticides are widely used to increase crop yields and vector control. However, both acute and chronic exposure have health consequences. There is paucity of information about the global occurrence of pesticide poisonings. Aim: To characterize the reports of pesticide exposures received by a University Poison Information Center. Material and Methods: All pesticide exposures reported in Chile between 2006 and 2013 were analyzed. A data-collection sheet provided by the International Programme on Chemical Safety of the World Health Organization, was used to collect information. Results: In the study period, 13,181 reports were analyzed. The main age groups exposed were preschoolers and adults. Sixty one percent of exposures occurred accidentally and $24.8 \%$ were suicide attempts. Exposures to acetylcholinesterase inhibitors was reported in $29.3 \%$ of cases, to superwarfarin rodenticides in $28.5 \%$ and to pyrethroids in $24.0 \%$. An increased risk of suicide attempts with pesticides was observed among women, when compared with men (odds ratio: 1.5; 95\% confidence intervals: 1.4-1.6; $p$ $<0.001$ ). The risk was higher among teenage girls. Conclusions: The amount of cases under acetyl cholinesterase inhibitor exposure, the most toxic pesticides currently in use should be highlighted. Workers should be educated and all cases should be reported to take actions aiming at reducing these events.

(Rev Med Chile 2015; 143: 1306-1313)

Key words: Pesticides; Poisoning; Toxic actions.
U n plaguicida o pesticida se puede definir como cualquier sustancia o mezcla de ellas destinadas a repeler, prevenir, controlar o combatir cualquier plaga, que pueda afectar al medioambiente, personas, animales, plantas $\mathrm{u}$ objetos inanimados ${ }^{1-2}$.

Se estima que a nivel mundial cada año se utilizan alrededor de 2,5 millones de toneladas de plaguicidas, cifra que va en aumento ${ }^{3}$. De esta can- tidad, Estados Unidos de Norteamérica da cuenta de $18 \%$ y Chile sólo de $1 \%$, que corresponde a 25.000 toneladas por año ${ }^{4}$.

Dentro de los beneficios que se pueden mencionar sobre el uso de plaguicidas, está el aumento del rendimiento de los cultivos. Se ha estimado que el uso de éstos previene la pérdida de 35 a 42\% de los cultivos y dado que la población mundial continúa en crecimiento y no existen recursos 
agrícolas ilimitados, al menos en la actualidad, no sería posible prescindir de ellos 5 .

A pesar de los indiscutibles beneficios que ha traído el uso de plaguicidas tanto en el área agrícola, sanitaria y veterinaria, hasta el día de hoy se utilizan algunos que son altamente tóxicos para los seres vivos y otros que han contaminado el medioambiente. Décadas atrás el extenso uso de los organoclorados, plaguicidas prohibidos internacionalmente por ser considerados contaminantes organopersistentes, afectó al medioambiente producto de su alta estabilidad y persistencia química. Debido a que estos compuestos tienen la cualidad de bioacumularse en las cadenas tróficas y pueden actuar como disruptores endocrinos, múltiples ecosistemas pudieron resultar afectados alterándose la fertilidad de algunas especies ${ }^{6,7}$.

Los riesgos asociados al uso de plaguicidas no sólo se relacionan con las alteraciones al ecosistema sino también a los seres humanos. Los plaguicidas son productos capaces de causar toxicidad ya sea por exposición aguda o crónica. Las intoxicaciones agudas pueden ser no intencionales o con intención suicida, siendo estas últimas las que presentan la mayor complejidad clínica. Por otro lado, la exposición crónica a éstos suele ser de carácter no intencional y es considerada un factor de riesgo para el desarrollo de enfermedades neurodegenerativas. Cabe mencionar que el impacto que tiene la exposición crónica a plaguicidas, en el aumento de la población enferma, es desconocido. Los principales grupos de riesgos son quienes los manipulan, aplican o quienes viven, trabajan o estudian cerca de zonas agrícolas, así como las mujeres embarazadas y niños en crecimiento que son familiares de trabajadores expuestos ${ }^{4}$.

Los datos disponibles hasta hoy por la Organización Mundial de la Salud (OMS) son muy limitados para conocer el impacto global de las intoxicaciones y muertes que se producen por plaguicidas $^{1,8}$. Sin embargo, se estima que 2.000 millones de personas trabajan y manipulan plaguicidas tanto en agricultura como en preservación de alimentos, y de éstos, 25 millones sufren una intoxicación ocupacional cada año. Además, un estudio estimó que aproximadamente $16 \%$ de las personas que trabajan en agricultura sufren alguna vez una intoxicación por plaguicidas ${ }^{3}$.

Datos de la American Association of Poison Control Centers muestran que durante 2010 a 2012 se recibieron aproximadamente 90.000 casos por año, de los cuales cerca de $94 \%$ fueron por exposición no intencional. De los plaguicidas involucrados, la principal familia fue la de piretrinas y piretroides, que dieron cuenta de casi $33 \%$ de los casos, los rodenticidas anticoagulantes de $11 \%$ y los inhibidores de la acetilcolinesterasa (I. AChE), es decir, organofosforados y carbamatos, de $6,5 \%{ }^{9-11}$.

A pesar de la importancia que tiene conocer el número de intoxicaciones por plaguicidas a nivel mundial, la mayoría de las veces sólo se pueden realizar estimaciones de las exposiciones agudas y de limitadas zonas geográficas. Dado lo anterior, el objetivo de este estudio es caracterizar las exposiciones agudas a plaguicidas registradas por el Centro de Información Toxicológica de la Pontificia Universidad Católica de Chile (CITUC).

\section{Material y Método}

Se realizó un estudio de tipo observacional descriptivo con componente analítico y retrospectivo. El universo de éste correspondió a todos los llamados recibidos por el CITUC desde Chile por una exposición o intoxicación con uno o más plaguicidas en humanos entre el período desde el 01 de enero de 2006 al 31 de diciembre de 2013.

La principal limitación de este estudio es que se excluyeron los brotes, que corresponden a dos o más pacientes expuestos en un mismo tiempo y lugar relacionados a un mismo plaguicida, debido a que no se contó con el número exacto de pacientes afectados. Lo anterior se debe a la gran dificultad que existe en contabilizar el número de pacientes $y$, en registrar simples datos como el sexo y la edad, pues con frecuencia éstos son desconocidos hasta resolver la intoxicación masiva. Además, en los brotes se observa una gran dispersión de pacientes dado que no todos son derivados a los mismos centros de salud lo que aumenta significativamente el riesgo de obtener múltiples casos repetidos.

La fuente de información utilizada correspondió a la base de datos de pacientes expuestos a plaguicidas del CITUC, que fue generada utilizando el programa INTOX Data Management System de la OMS. Los datos fueron recopilados utilizando el instrumento validado elaborado por The International Programme on Chemical Safety (IPCS) INTOX de la OMS ${ }^{12}$. Por cada llamado 
que recibe el CITUC, el profesional que lo atiende debe completar este instrumento en papel que posteriormente es ingresado a la base de datos electrónica desde donde se extrajo la información.

Las variables analizadas fueron: edad (Neonato: $<29$ días, lactante: 30 días a 11 meses, preescolar: 1 a 4 años, escolar: 5 a 14 años, adolescente: 15 a 19 años, adulto: 20 a 64 años, anciano: $\geq 65$ años); sexo; familia del plaguicida (inhibidor de la acetilcolinesterasa, rodenticida, piretroide, herbicida, fungicida, otros); circunstancia (accidental, suicida, ocupacional, otras); vía de exposición (ingestión, inhalación, dérmica, otras) y mes.

Para el análisis estadístico, los datos fueron tabulados en Microsoft ${ }^{\circledR}$ Excel 2010. Éste se realizó utilizando el programa STATCALC ${ }^{\circledR}$ 6.0.1 e IBM SPSS ${ }^{\circledR}$ 20.0. Se obtuvieron porcentajes para las variables categóricas, se identificaron factores y grupos de riesgo a través del Odds Ratio (OR).

\section{Resultados}

Durante el período entre 2006 y 2013, CITUC recibió 227.895 llamados, de los cuales 15.323 se relacionaron con plaguicidas (6,7\%). De éstos, se eliminaron las consultas no toxicológicas, los brotes, los casos ocurridos en el extranjero y aquellos en animales resultando un total de 13.181 casos incluidos en este estudio.

$\mathrm{Al}$ analizar las características del paciente, sexo y grupo etario (Tabla 1), se observa que $55,4 \%$ de los casos correspondió a pacientes de sexo mas- culino. Los principales grupos etarios correspondieron a pacientes adultos $(47,3 \%)$ y preescolares $(31,3 \%)$ donde el rango de edad varió desde 5 días a 95 años con una mediana de 20 años. Por otro lado, al analizar la circunstancia de exposición, que se observa en la Figura 1, se obtuvo que $60,8 \%$ de los casos se produjo por exposiciones de tipo accidental y $24,8 \%$ por intención suicida.

Las exposiciones a plaguicidas pueden ocurrir por uno o múltiples productos. Con respecto a la familia del plaguicida, en la Figura 2 se observa que, de los pacientes expuestos a sólo un plaguicida (97,5\% de los casos), los I. AChE, los rodenticidas superwarfarínicos y los piretroides causaron 29,$3 ; 28,5$ y $24,0 \%$ de las exposiciones, respectivamente. Y, de los I. AChE, los organofosforados predominaron con $86,1 \%$ de los casos. Por otra parte, respecto a la vía de exposición (Figura $3)$, los pacientes que se expusieron por vía única representaron $86,7 \%$ de los casos totales, principalmente por vía digestiva $(73,8 \%)$ e inhalatoria $(15,6 \%)$.

Al realizar un análisis de riesgo entre la familia del plaguicida y el grupo etario destacó que en los pacientes pediátricos predominó la ingesta de rodenticidas. Al relacionar el grupo etario (pediátrico; otros grupos etarios) y el plaguicida (rodenticidas; otros plaguicidas) se observa que estos pacientes presentaron un riesgo 4 veces mayor de sufrir una exposición a un rodenticida (OR: 4,$\left.16 ; \mathrm{IC}_{95 \%}: 3,83-4,52 ; \mathrm{p}<0,001\right)$ en comparación con otro plaguicida (Tabla 2). Se consideró sólo como paciente pediátrico a los menores de 6 años.

Tabla 1. Distribución de casos según grupo etario y sexo. $n=13.181$

\begin{tabular}{|c|c|c|c|c|}
\hline \multirow{3}{*}{$\begin{array}{l}\text { Grupo etario } \\
\text { Neonatos (< } 29 \text { días) }\end{array}$} & \multicolumn{3}{|c|}{ Sexo } & \multirow[t]{2}{*}{ Total } \\
\hline & $\mathbf{M}$ & $\mathbf{F}$ & Desconocido & \\
\hline & $7 \quad(0,1 \%)$ & $3(0,0 \%)$ & $0(0,0 \%)$ & $10 \quad(0,1 \%)$ \\
\hline Lactantes (30 días a 11 meses) & $121 \quad(0,9 \%)$ & $75 \quad(0,6 \%)$ & $4(0,0 \%)$ & $(1,5 \%)$ \\
\hline Preescolares (1 a 4 años) & $2.370(18,0 \%)$ & $1.739(13,2 \%)$ & $12(0,1 \%)$ & $4.121 \quad(31,3 \%)$ \\
\hline Escolares (5 a 14 años) & $467 \quad(3,5 \%)$ & $455 \quad(3,5 \%)$ & $3(0,0 \%)$ & $925 \quad(7,0 \%)$ \\
\hline Adolescentes (15 a 19 años) & $384 \quad(2,9 \%)$ & $430 \quad(3,3 \%)$ & $0(0,0 \%)$ & $814 \quad(6,2 \%)$ \\
\hline Adultos (20 a 64 años) & $3.490(26,5 \%)$ & $2.735(20,7 \%)$ & $9(0,1 \%)$ & $6.234(47,3 \%)$ \\
\hline Anciano (> 65 años) & $238 \quad(1,8 \%)$ & $186(1,4 \%)$ & $0(0,0 \%)$ & $424 \quad(3,2 \%)$ \\
\hline Desconocido & $229(1,7 \%)$ & $178(1,4 \%)$ & $46(0,3 \%)$ & $(3,4 \%)$ \\
\hline Total & $7.306(55,4 \%)$ & $5.801(44,0 \%)$ & $74(0,6 \%)$ & $13.181(100,0 \%)$ \\
\hline
\end{tabular}

M: Masculino; F: Femenino. 

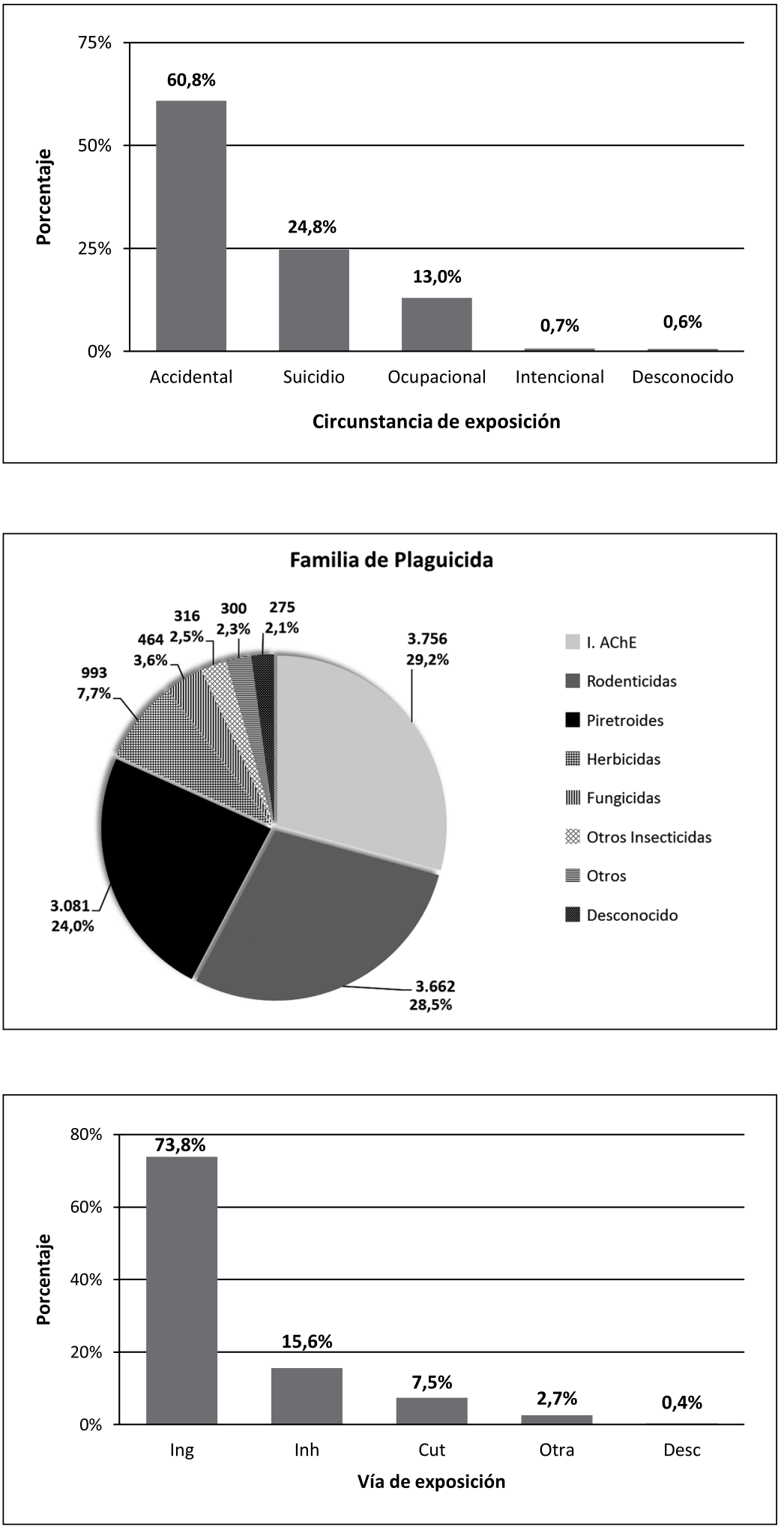

Figura 1. Distribución de casos según circunstancia de exposición. $\mathrm{n}=13.181$.

Figura 2. Distribución de casos expuestos a 1 solo agente según familia del plaguicida. $n=12.847$. I. AChE: Inhibidor de la acetilcolinesterasa.

Figura 3. Distribución de casos según vía de exposición única. $\mathrm{n}=$ 11.434. Ing: Ingestión; Inh: Inhalatoria; Cut: Cutánea; Desc: Desconocida. 
En la Figura 4 se observa el comportamiento de los llamados recibidos según mes donde se aprecia existencia de un mayor número de intoxicaciones ocurridas en los meses cálidos en comparación con los meses fríos.
Durante 2006 a 2013 se recibieron 3.263 casos por intento de suicidio con plaguicidas. En la Tabla 3 se observa que los grupos etarios en que predominó esta circunstancia fueron los adultos y los adolescentes. En los adultos predominaron

Tabla 2. Riesgo de exposición a rodenticidas por pacientes pediátricos

\begin{tabular}{|lccccc|}
\hline Grupo etario & Ingesta de rodenticida & Exposición a otro plaguicida & OR & IC95\% & Valor $\mathbf{p}$ \\
\hline Paciente pediátrico & 2.188 & 2.395 & 4,16 & $3,83-4,52$ & $<0,001$ \\
\hline Paciente no pediátrico & 1.418 & 6.461 & & \\
\hline
\end{tabular}

Se excluyeron 719 casos los cuales tenían grupo etario o plaguicida desconocido.

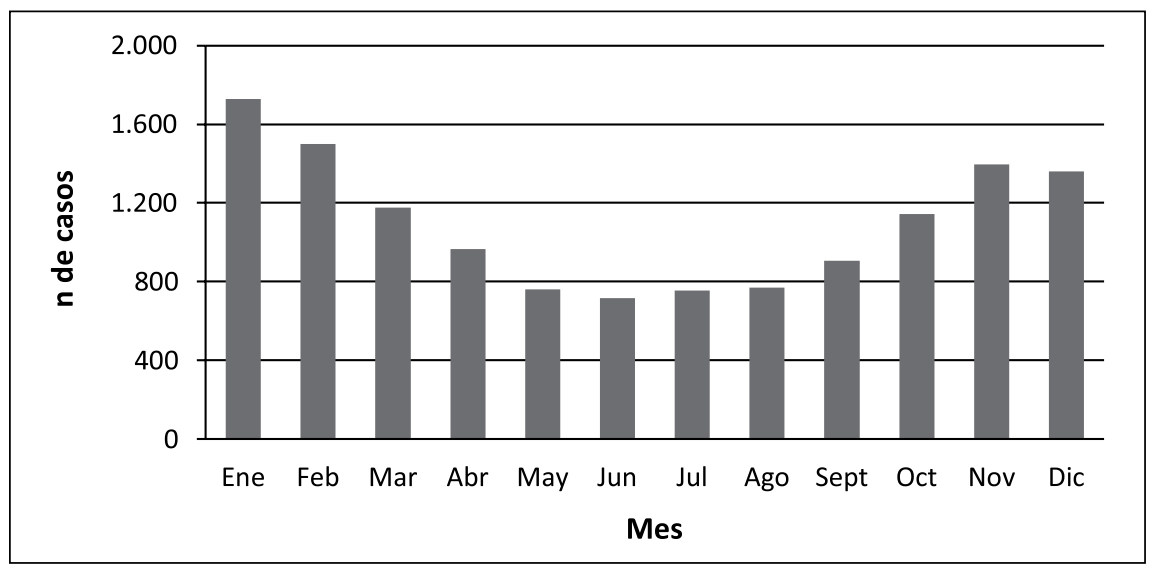

Figura 4. Distribución de casos según mes. $n=13.181$.

Tabla 3. Riesgo de intento de suicidio según grupo etario y sexo

\begin{tabular}{|c|c|c|c|c|c|}
\hline Grupo etario/circunstancia & Suicida & No suicida & OR & $I_{95 \%}$ & Valor-p \\
\hline Escolar & 129 & 784 & \multirow[b]{3}{*}{4,68} & \multirow[b]{3}{*}{$2,94-7,52$} & \multirow[b]{3}{*}{$<0,001$} \\
\hline $\mathrm{F}$ & 102 & 350 & & & \\
\hline M & 27 & 434 & & & \\
\hline Adolescente & 523 & 287 & \multirow[b]{3}{*}{3,57} & \multirow[b]{3}{*}{$2,61-4,90$} & \multirow[b]{3}{*}{$<0,001$} \\
\hline $\mathrm{F}$ & 334 & 95 & & & \\
\hline $\mathrm{M}$ & 189 & 192 & & & \\
\hline Adulto & 2.446 & 3.735 & \multirow[b]{3}{*}{1,29} & \multirow[b]{3}{*}{$1,17-1,44$} & \multirow[b]{3}{*}{$<0,001$} \\
\hline $\mathrm{F}$ & 1.169 & 1.548 & & & \\
\hline M & 1.277 & 2.187 & & & \\
\hline Anciano & 92 & 326 & \multirow[b]{3}{*}{0,72} & \multirow[b]{3}{*}{$0,44-1,19$} & \multirow[b]{3}{*}{ NS } \\
\hline $\mathrm{F}$ & 35 & 150 & & & \\
\hline M & 57 & 176 & & & \\
\hline Desconocido & 66 & 335 & & & \\
\hline Total general & 3.256 & 9.770 & \multirow{3}{*}{1,46} & \multirow{3}{*}{$1,35-1,58$} & \multirow{3}{*}{$<0,001$} \\
\hline $\mathrm{F}$ & 1.672 & 4.099 & & & \\
\hline M & 1.584 & 5.671 & & & \\
\hline
\end{tabular}

F: Femenino, M: Masculino, OR: Odds Ratio, IC: Intervalo de Confianza. NS: No significativo. 73 tenían sexo o grupo etario desconocido, por lo que éstos fueron excluidos del análisis segmentado. Para analizar el total general, sólo se excluyeron aquellos que tenían sexo desconocido (4 casos). 
los casos por pacientes de sexo masculino mientras que en los adolescentes, aquellos de sexo femenino. Al realizar un análisis según circunstancia y sexo se observó que las pacientes de sexo femenino presentaron un riesgo 1,46 veces mayor al intento de suicidio, en comparación al sexo masculino. Éste varió en los distintos grupos etarios donde las escolares, adolescentes y mujeres adultas presentaron un riesgo de 4,7; 3,6 y 1,3 veces mayor, respectivamente.

\section{Discusión}

Al igual que lo observado en la Tabla 1 con respecto al sexo de los pacientes, diferentes estudios epidemiológicos han mostrado el predominio de casos de pacientes de sexo masculino en comparación a los de sexo femenino, cuando el agente causal es un plaguicida ${ }^{13,14}$. Datos ministeriales muestran que, en Chile entre 2006 y 2012, 2.339 hombres y 1.934 mujeres sufrieron una intoxicación por plaguicidas, misma tendencia se observa desde $1997^{13}$. Por otro lado, con respecto a la distribución observada para el grupo etario (Tabla 1), esta es similar a la mostrada por estudios realizados en Asia y Latinoamérica. En cambio, según reportes de Estados Unidos de Norteamérica son los pacientes pediátricos quienes más se exponen a plaguicidas, y en segundo lugar los adultos ${ }^{11,14,15}$. Es decir, esta tendencia varía según país.

Con respecto a la circunstancia de exposición (Figura 1), la distribución observada concuerda con la realidad tanto a nivel nacional como internacional, donde las exposiciones no intencionales, ya sean accidentales u ocupacionales, predominan sobre las de intencionalidad suicida ${ }^{11,13,14}$.

En relación a la familia de plaguicida causal, a nivel internacional Estados Unidos de Norteamérica mostró que los piretroides dan cuenta de $35,2 \%$ de las exposiciones, los rodenticidas superwarfarínicos de $10,8 \%$ y los I. AChE de $6,1 \%{ }^{11}$. En Corea son los herbicidas y fungicidas los que dan cuenta de la mayoría de los $\operatorname{casos}^{14}$, mientras que en Ecuador $71,4 \%$ de las exposiciones son por I. $\mathrm{AChE}^{15}$. De estos estudios se observó que la exposición a plaguicidas, es altamente variable en los distintos países y, por lo tanto, resulta difícil establecer una tendencia comparable con la realidad de Chile. A nivel país, los I. AChE son los que dan cuenta del mayor número de casos en comparación con los piretroides ${ }^{13}$. Lo anterior se debe a que en Chile sólo se considera como caso a aquellos pacientes que presentaron síntomas, por lo que se espera que los I. AChE predominen ya que son de mayor toxicidad para los mamíferos. A pesar de lo anterior, la tendencia mostrada es similar a la observada en la Figura 2. Es decir, no sólo producen la mayor cantidad de exposiciones sino también de intoxicaciones. Lo anterior deja en evidencia que en Chile se utilizan plaguicidas de mayor toxicidad para los humanos en comparación con Estados Unidos de Norteamérica y esto es un evidente riesgo para la salud de quienes se exponen.

En relación a la vía de exposición (Figura 3) se observó predominio de la vía oral. Dado que esta resulta natural y poco invasiva suele ser la principal en exposiciones accidentales y suicidas, circunstancias que también dieron cuenta de la mayor cantidad de casos. Por el contrario, en las exposiciones ocupacionales, las vías de exposición que destacan son la inhalatoria y cutánea, principalmente por la falta del uso de elementos de protección personal o incumplimientos del período de reingreso ${ }^{4}$.

Como se observó en la Tabla 2, los pacientes pediátricos dieron cuenta del mayor número y riesgo de exposición a rodenticidas. Lo anterior podría deberse al almacenamiento de plaguicidas al alcance de los niños, y en el caso específico de los rodenticidas, su aplicación en lugares donde los niños circulen pues suelen confundirlos e ingerirlos. Lo anterior es relevante dado que sólo los menores de 6 años corresponden a $35,2 \%$ de los pacientes estudiados.

Con respecto al número de casos relacionados con plaguicidas según mes (Figura 4), la tendencia observada es la misma que a nivel país durante 2007 a 2011. Y además, datos de 2012 también mostraron la disminución de casos en otoño e invierno, en comparación al verano ${ }^{13}$. El mayor número de exposiciones en los meses de verano podría explicarse debido al peak de la temporada agrícola y por las variaciones de insectos según temporada, que tienen un máximo en primavera verano y un mínimo en otoño invierno ${ }^{16}$.

En la Tabla 3 se observa el número casos y riesgo de intento de suicidio según grupo etario y sexo, donde se aprecia que los adultos fueron el grupo que dio cuenta de la mayor cantidad de intentos de suicidio, comportamiento que ha sido 
observado tanto a nivel nacional e internacional ${ }^{4,14}$. Esto se debe a que los adultos son la población con mayor acceso a estos productos $y$, en algunos casos, trabajan directamente con ellos.

La presencia de casos en el grupo etario de los escolares se explica debido a que este incluye pacientes hasta los 14 años donde el intento suicida se reporta desde los 11 años. Es más, datos internacionales muestran que las exposiciones intencionales se inician desde los 12 años, una edad que concuerda con la observada ${ }^{11}$. Por otro lado, datos a nivel nacional también han registrado intentos de suicidio de pacientes de corta edad donde el menor de ellos tenía 12 años ${ }^{13}$. Lamentablemente, estos casos no han sido descritos extensamente por lo que no existen estadísticas que permitan realizar mayor comparación. El alto riesgo de intento de suicidio observado para el sexo femenino en todos los grupos etarios, con excepción de los ancianos, nunca había sido observado anteriormente cuando el agente era plaguicida tanto a nivel nacional como internacional. Lo anterior, hace imperativa la necesidad de realizar tests que permitan detectar tempranamente aquellos factores de riesgos y determinantes sociales que conlleven a los intentos de suicidio, con la finalidad de implementar estrategias preventivas para abarcar este problema.

Un dato alarmante, fue que el riesgo de suicidio en escolares obtenido en este estudio fue mayor al de los adolescentes, sin embargo, estos dieron cuenta de un mayor número de casos. Cabe mencionar que la principal circunstancia de exposición en adolescentes es el intento de suicidio, por lo que se hace énfasis en que cada vez que se reciba a un paciente menor de edad con posible intencionalidad suicida este reciba el apropiado apoyo e interconsulta psiquiátrica.

En conclusión, se describió el perfil de las exposiciones según paciente, plaguicida, vía de exposición y temporada del año. Al describir los casos de pacientes con intencionalidad suicida, se observó riesgo de intento de suicidio para el sexo femenino en todos los grupos etarios, con excepción de los ancianos. Se observó predominio de las exposiciones por I. AChE, dado lo anterior se destaca la importancia de educar a los trabajadores sobre la correcta manipulación de plaguicidas con el fin de evitar intoxicaciones y a la población general acerca del almacenamiento, sobre todo en presencia de pacientes pediátricos. Además, se hace hincapié en la necesidad de, cada vez que se reciba un caso por exposición a plaguicidas, notificarlo a la autoridad sanitaria correspondiente, que a futuro permitirá tomar acciones como, por ejemplo, restringir el uso de aquellos plaguicidas altamente tóxicos. Y para terminar, es importante recalcar la necesidad de realizar estudios para determinar el impacto que tiene la exposición crónica a plaguicidas en el aumento de desarrollo de enfermedades neurodegenerativas.

\section{Referencias}

1. WHO, Guidelines on Public Health Pesticide Management Policy. Pesticide Evaluation Scheme, Department of Control of Neglected Tropical Diseases, Geneva (2010). Disponible en http://www.who.int/whopes/resources/ SEA_CD_214.pdf [Consultado el 10 de enero de 2015].

2. Decreto Supremo $N^{\circ} 88$, Artículo 1 y 2, Reglamento de Notificación Obligatoria de las Intoxicaciones con Pesticidas. 17 de mayo de 2004. Publicado en el diario oficial el 05 de octubre de 2004. Santiago, Chile.

3. Alavanja M. Pesticides Use and Exposure Extensive Worldwide. Rev Environ Health 2009; 24 (4): 303-9.

4. Vallebuona C, Solar O, Grau P, Suárez S, Concha C, Winser ME, et al. Norma técnica de vigilancia de intoxicaciones agudas por plaguicidas. División de planificación Sanitaria, Departamento de Epidemiología, Ministerio de Salud, Gobierno de Chile (2007).

5. Zhang W, Jiang F, Ou J. Global pesticide consumption and pollution: with China as a focus. Proceedings of the International Academy of Ecology and Environmental Sciences, 2011; 1 (2): 125-44.

6. World Health Organization. Pesticides. 2008. Disponible en: http://www.who.int/ceh/capacity/Pesticides.pdf [Consultado el 10 de enero de 2015].

7. Ritter L, Solomon KR, Forget J. Persistent Organic Pollutants. The International Programme on Chemical Safety. Disponible en: http://www.chem.unep.ch/pops/ ritter/en/ritteren.pdf [Consultado el 10 de enero de 2015].

8. Thundiyil JG, Stober J, Besbelli N, Pronczuk J. Acute pesticide poisoning: a proposed classification tool. Bull World Health Organ 2008; 86 (3): 205-9.

9. Bronstein AC, Spyker DA, Cantilena LR, Green JL, Rumack BH, Dart RC. 2010. Annual Report of the American Association of Poison Control Centers' National Poison Data System (NPDS): 28th Annual Report. Clin Toxicol, 2011; 49 (10): 910-41.

10. Bronstein AC, Spyker DA, Cantilena LR, Rumack BH, Dart RC. 2011 Annual report of the American Associa- 
tion of Poison Control Centers' National Poison Data System (NPDS): 29th Annual Report. Clin Toxicol, 2012; 50 (10): 911-1164.

11. Mowry JB, Spyker DA, Cantilena LR, Bailey JE, Ford M. 2012. Annual Report of the American Association of Poison Control Centers' National Poison Data System (NPDS): 30th Annual Report. Clin Toxicol, 2013; 51 (10): 949-1229.

12. Paris E, Ríos JC. INTOXICACIONES. Epidemiología, Clínica y Tratamiento. Santiago, Chile; Ediciones Pontificia Universidad Católica de Chile; 2005. pp. 24-5.

13. Informe Vigilancia Intoxicaciones Agudas por Plaguicidas. Departamento de Epidemiología, Ministerio de
Salud, Gobierno de Chile. 2012. Disponible en: http:// epi.minsal.cl/epi/0notransmisibles/vent-revep/REVEP_2012.pdf [Consultado el 10 de enero de 2015].

14. Cha ES, Khang YH, Lee WJ. Mortality from and Incidence of Pesticide Poisoning in South Korea: Findings from National Death and Health Utilization Data between 2006 and 2010. PLoS one 2014: 9 (4): e95299.

15. González F, López R, Estévez E. Acute pesticide poisoning in Ecuador: a short epidemiological report Journal of Public Health, 2010; 18 (5): 437-42.

16. Nicholls C. Control biológico de insectos: un enfoque agroecológico. Medellín, Colombia. Editorial Universidad de Antioquia. 2008. pp. 159-205. 